\title{
Plasmapause Morphology Determined From an Empirical Ionospheric Convection Model
}

\author{
Richard A. Doe, Mark B. Moldwin , AND Michael Mendillo
}

Center for Space Physics and Department of Astronomy, Boston University, Boston, Massachusetts

\begin{abstract}
The steady state electric potentials derived from empirical ionospheric convection models were mapped to the magnetospheric equatorial plane and added to the Earth's rotational field to examine the local time dependence of the plasmapause radius. The last closed equipotential line was assumed to be the plasmapause. The sensitivity of plasmapause shape and size derived in this way was studied by varying the $z$ and $y$ components of the interplanetary magnetic field (IMF) and the geomagnetic activity index Kp. Results show that the Heppner and Maynard model produces plasmasphere morphology that is broadly consistent with prior ground-based and satellite measurements of plasmapause position. Our model results show that the largest change in the characteristic shape of the plasmapause is due to the direction of IMF $B_{z}$; for IMF $B_{z}$ southward, the plasmapause is circular, while a broad oval plasmapause (with a bulge at 2100 UT) is found for IMF $B_{z}$ northward. The ability of our model to reproduce the $L_{p p}$-LT shape of the plasmapause as found by previous observations shows that empirical ionospheric convection models can be extended to provide a useful method to determine plasmapause location for a variety of steady geomagnetic conditions.
\end{abstract}

\section{INTRODUCTION}

The location and shape of the plasmapause have been modeled by Nishida [1966], Brice [1967], and Mendillo and Papagiannis [1971] by adopting a value for the dawn-dusk convection electric field induced across the magnetosphere by the solar wind and adding this to the Earth's rotational electric field. For these "convection-plasmapause" models, the last closed potential "streamline" defines the plasmapause. The results of these models give basically symmetric, teardrop-shaped plasmapauses with a bulge around dusk (cf. Figure 1). Chen and Wolf [1972] refined this approach by including a time-varying convection electric field to produce dynamic, asymmetric shapes with complex bulge regions. The validity of the assumption that the plasmapause is coincident with the flow equipotential has been challenged by workers such as Carpenter [1970] on the basis of the time scales required for the plasmapause to reach a particular equilibrium configuration. Park [1970] determined that an "empty" magnetospheric flux tube at about $L=$ 5 takes on the order of days to refill, casting doubt on the coincidence of the flow equipotential and the sharp density ledge classically defined as the plasmapause. However, for extended periods of steady geomagnetic activity, the plasmapause will approach such an equipotential [Carpenter, 1970]. Another factor raised in questioning the validity of assuming the plasmapause is a flow equipotential was the highly nonuniform distribution of convection electric fields. In the earlier models, a constant dawn-dusk convection electric field which was assumed to penetrate throughout the magnetosphere was used. This becomes a poor approximation to the magnetospheric convection electric field, particularly during substorms, when the electric field becomes highly nonuniform. Also, the assumption of the ability of the convection electric field to penetrate throughout the magnetosphere

Copyright 1992 by the American Geophysical Union.

Paper number 91JA01649.

0148-0227/92/91JA-01649\$05.00 is another issue that has been raised about the validity of the flow equipotentials representing the plasmapause [Southwood and Wolf, 1978].

Observations of the $L$-LT shape and size of the plasmapause under various geomagnetic conditions have been investigated by several workers [e.g., Carpenter, 1966; Taylor et al., 1969; Chappell et al., 1971; Maynard and Grebowsky, 1977; Horwitz et al., 1990] using a variety of ground-based and satellite techniques. They all found a plasmapause that decreases in radius for increased geomagnetic activity, and all show the existence of a bulge located within \pm 3 hours around dusk. The average results of Carpenter [1966], Chappell et al. [1971], and Maynard and Grebowsky [1977], as well as a fit to the Horwitz et al. [1990] DE data, are shown in Figure 2. Note that Carpenter's classic pattern and the Maynard and Grebowsky shape fix the average location of the bulge in the postdusk region and are characterized by a generally circular shape at all other local times. Chappell et al. found a bulge symmetric about dusk, and Horwitz et al. found an afternoon bulge; both of their results can be characterized as large oval-shaped plasmapauses. The clear difference in previously published plasmapause shapes may be attributed to differences in measurement technique and $L$ shell range of observations as well as varying interplanetary magnetic field (IMF) and geomagnetic conditions.

The dynamics of the bulge have also been studied during periods of varying geomagnetic activity. Carpenter [1970] found an afternoon bulge for prolonged periods of high $\mathrm{Kp}$ and a postdusk bulge during quiet times. This was attributed to the ability of the magnetospheric convection flow to penetrate into the dayside for high geomagnetic activity, while corotation flow dominated for quiet times.

In this study, we examine the results of mapping the convection electric potential at ionospheric heights, as derived from empirical ionospheric convection models, to the equatorial plane in the magnetosphere where it is added to the Earth's rotational potential. The ionospheric convection model of Heppner and Maynard [1987] was used as an initial test case. Other empirical convection models can 


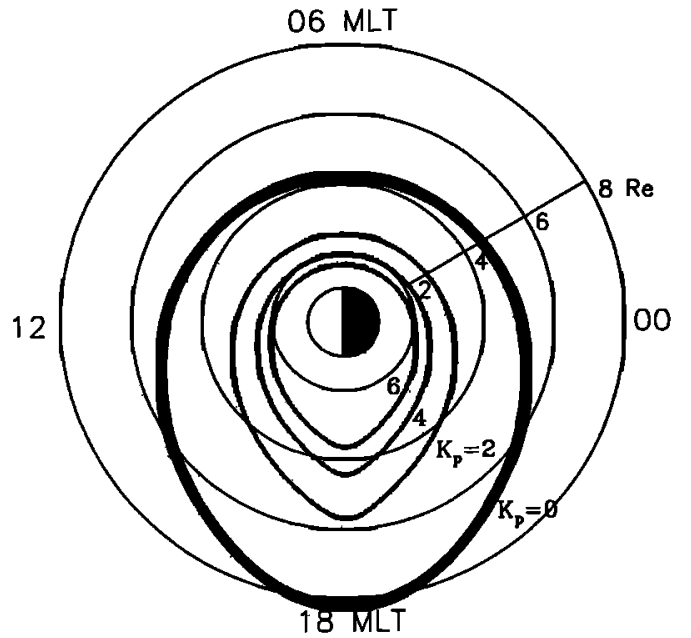

Fig. 1. Results derived from a constant dawn-dusk electric field [cf. Grebowsky, 1970] to represent the solar wind induced magnetospheric convection electric field. Note the symmetric "teardrop". shaped plasmapauses that vary in size depending on geomagnetic activity.

be used, such as those of Heelis et al. [1982] and Holt et al. [1987], but are not as easily parameterized in terms of geomagnetic indices or IMF direction. The input parameters for the Heppner and Maynard model are IMF $B_{z}$ and $B_{y}$ direction and the geomagnetic index $\mathrm{Kp}$. The resultant "equipotential plasmapause" then becomes a function of the convection modeling parameters, thereby allowing a study of the morphology of the plasmapause with respect to IMF, a feature that has not been examined in previous studies. Moreover, since all plasmapause measurements to date have been organized by $\mathrm{Kp}$, use of the Heppner and Maynard model allowed comparison of model results derived from convection patterns directly with plasmapause observations.

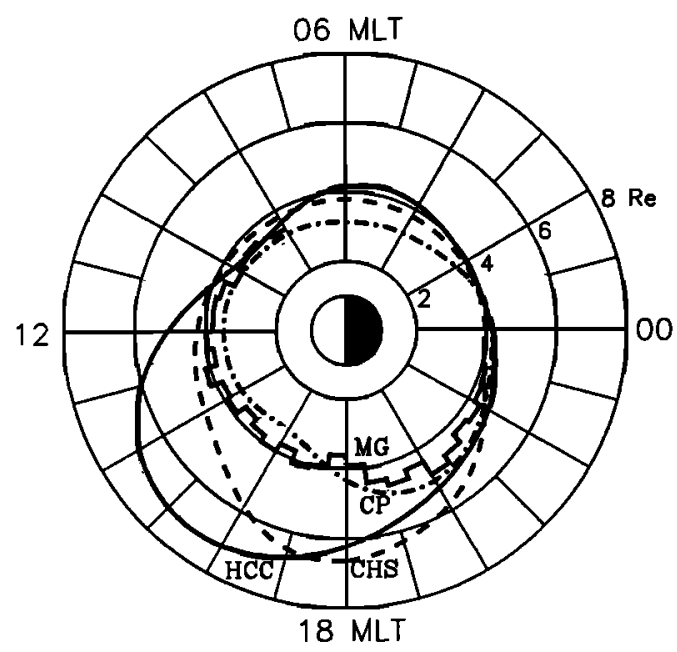

Fig. 2. Comparison of average plasmapanse loci from published results. Carpenter [1966] (CP) derived the shape from whistler data, Chappell et al. [1971] (CHS) used OGO$5 \mathrm{H}^{+}$density gradients, and Maynard and Grebowsky [1977] (MG) used Explorer 45 dc probe saturations to determine their plasmapause. The plasmapanse labeled HCC is a third harmonic fit to the DE 1 RIMS low-energy ion transition data gathered for Kp $=0$ to 6 by Horwitz et al. [1990].

\section{MODEL Description}

Following the early suggestions of Nishida [1966] and Brice [1967], we define the plasmapause as the last closed equipotential line in the equatorial plane. The input ionospheric convection model provides the electric potential in kilovolts at every hour of magnetic local time (MLT) and every degree of invariant latitude from $51^{\circ}$ to $88^{\circ}$. The resulting 24 by 38 element array is interpolated and expanded to a larger square array ( 512 by 512 elements) in order to provide sufficient resolution for magnetospheric mapping. This "smoothed" potential field is then entered into a target array through conversion of invariant latitude to $L$ shell by means of a dipole field mapping. For each $L$ shell radius in the geocentric solar magnetospheric (GSM) equatorial plane, the Earth's rotational potential is given by

$$
\Phi_{\text {rot }}(L)=\frac{-\omega B_{s}\left(R_{E}\right)^{2}}{L}=-\frac{88.75}{L} \mathrm{kV}
$$

where $\omega$ is the angular rotation of the Earth, $L$ is the radial distance measured in earth radii $R_{E}$, and $B_{s}$ is the surface dipole field at the equator [see Hones and Bergeson, 1965]. This rotational potential in kilovolts is simply added to the convection potential at every $L$ shell and MLT in the target array. The array is then examined for the largest closed equipotential by an iterative contouring routine.

A sample target array that shows the combined effect of the earthward magnetospheric convection and the azimuthal corotation flow is shown in Figure $3 a$ for $\mathrm{Kp}=3, B_{z}$ southward and $B_{y}$ positive. This figure shows the magnetospheric flow lines in the equatorial plane. No magnetopause is included in this figure, but the flow pattern is reminiscent of that drawn by Nishida [1966], who was the first to examine the effect of convection on the shape and size of the plasmapanse. The last closed equipotential line is highlighted and corresponds to the plasmapause location.

Figure $3 b$ shows the input ionospheric convection potential given by the Heppner and Maynard model for the case depicted in Figure $3 a$. This potential is shown in its original MLT versus invariant latitude "polar" grid. Aside from the total voltage drop across the polar cap, this specific $(\mathrm{Kp}=3)$ pattern is very similar to that for $K p=0$ and $K p=6$. The boundaries at " $A$ " and " $B$ " mark the mean polar location of the $K p=0$ and $K p=6$ plasmapauses mapped back to the polar cap, respectively. Notice that this "interaction region" (i.e., the region where convection and corotation have roughly equal influence) spans the shallow slopes of the potential pattern and excludes the high-latitude maximum and minimum. Although most of the detailed structure in the Heppner and Maynard model is poleward of this interaction region, we assume its validity extends to the lower-latitude $\left(\Lambda=51^{\circ}\right)$ limit of the model. The location of the interaction region at relatively low latitudes will allow the use of other empirical ionospheric convection models, such as the Millstone Hill incoherent scatter radar convection model [Holt et al., 1987], which have limited latitudinal coverage but include this region.

The approach of using the empirical ionospheric electric potentials to determine the plasmapause morphology eliminates the need to determine the effect of ring current shielding on magnetospheric convection since this effect is implicitly included in observationally based ionospheric convection models. However, brief periods of enhanced convection may have a significant effect on plasmapause morphology, and 


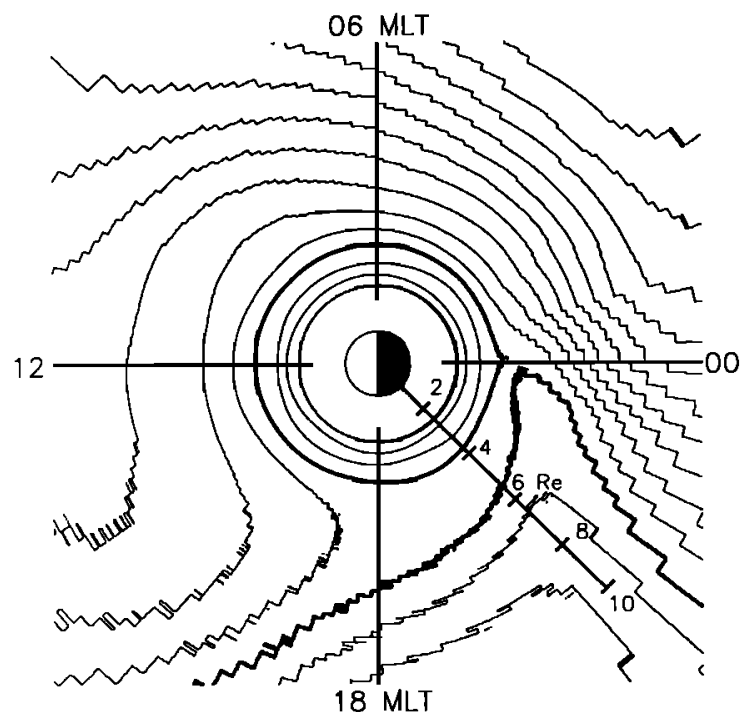

(a)

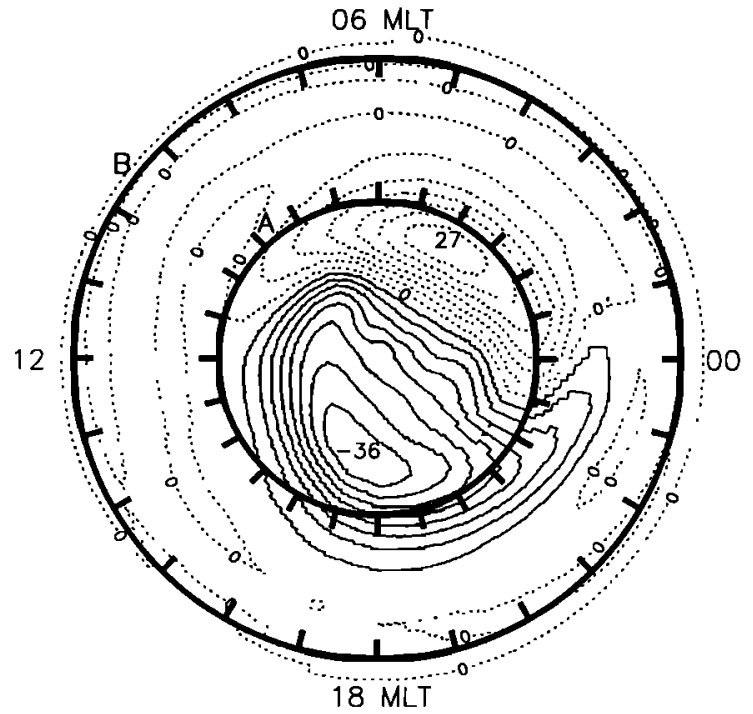

(b)

Fig. 3. (a) The magnetospheric convection pattern determined from the results of our ionospheric convection potential mapping technique using the Heppner and Maynard [1987] model results. The last closed equipotential is highlighted. (b) The Heppner and Maynard convection pattern plotted in the northern polar cap for IMF $B_{z}$ southward, $B_{y}$ positive and $K_{p}=3$. The plasmapause is found equatorward of the potential maximum and minimum and is located between the boundaries $A$ and $B$. Boundaries $A$ and $B$ indicate the mean plasmapause location for $K p=0$ and $K p=6$, respectively.

this effect will not be produced with time-averaged convection models.

\section{RESULTS AND DISCUSSION}

\section{Plasmapause Morphology and Location of the Bulge}

Figures $4 a$ and $4 b$ summarize the IMF $B_{z}$ southward plasmapause shapes determined from our convection potential mapping technique for different $\mathrm{Kp}$ values and IMF $\boldsymbol{B}_{\boldsymbol{y}}$ orientations. A close inspection of Figure 2 indicates that the modeled plasmapause shapes are more consistent with the Carpenter [1970] and Maynard and Grebowsky [1977] shapes than the Chappell et al. [1971] and Horwitz et al. [1990] shapes. That is, they are nearly circular with a very small premidnight bulge rather than oval with a broad symmetric bulge. The bulge in our model is very small, less than $\frac{1}{2} R_{E}$ from the mean radius. However, upon close examination of Figure $3 a$, we see that the postdusk region is where the corotating plasma is counterstreaming against the earthward convecting magnetospheric plasma. Thus the stagnation point, or bulge region, lies in this local time sector. The stagnation point is the place where the flow due to corotation is balanced by magnetospheric convection. In previous models [e.g., Nishida 1966; Mendillo and Papagiannis, 1971], the applied dawn-dusk electric field implied a sunward convection flow from the magnetotail (i.e., flow directed from 2400 to $1200 \mathrm{LT}$ ). This will always give the stagnation point in the flow at dusk. The Heppner and Maynard [1987] model gives an asymmetric flow pattern that appears to be greatly influenced by the Harang discontinuity, or the locus of points in the nightside auraral zone across which the meridional component of the ionospheric electric field reverses from a basically poleward field to a basically equatorward field [Erickson et al., 1991]. This reversal is evident in Figure $3 a$ on the nightside. This effect gives a stagnation point between dusk and midnight depending on the strength of the magnetospheric convection electric field (i.e., the polar cap potential drop). Detailed comparisons of our modeled bulge locations with prior results are limited by the relatively small number of reported bulge observations.

\section{Plasmapause Morphology for IMF $B_{z}$ northward}

Unlike ionospheric convection models with IMF $B_{z}$ southward, the $B_{z}$ northward models are widely disparate as individual researchers struggle to fit finely structured convection features into a global pattern. The Heppner and Maynard model adopts the paradigm of a distorted two-cell pattern for IMF $B_{z}$ slightly northward and accepts IMF $B_{y}$ as a shapecontrolling input parameter. No provision is made for modulation by geomagnetic $\mathrm{Kp}$ index. Furthermore, the Heppner and Maynard model for IMF $B_{z}$ strongly northward suggests a splitting of the two-cell pattern into a weak three-cell pattern. The plasmapause shape and extent are shown in Figure $4 c$ for IMF $B_{z}$ slightly northward and both IMF $B_{y}$ orientations. Little difference in plasmapause size and shape is indicated for these cases or for the IMF $B_{z}$ strongly northward cases shown in Figure $4 d$. This suggests that while the higher-latitude potential field is very sensitive to the strength of IMF $B_{z}$, lower-latitude convection remains constant in the plasmapause "interaction region." This is consistent with the view that the higher-latitude (variable) potential is a consequence- of $B_{z}$ modulated merging/reconnection while the lower-latitude (steady) convection potential is a result of viscous momentum transport [cf. Reiff et al., 1981]. The biggest difference (over $1 R_{E}$ ) is due to changing the IMF $B_{y}$ direction for IMF $B_{z}$ strongly northward.

The morphologies of the IMF $B_{z}$ northward plasmapauses are very similar to the oval shaped broad bulged plasma- 

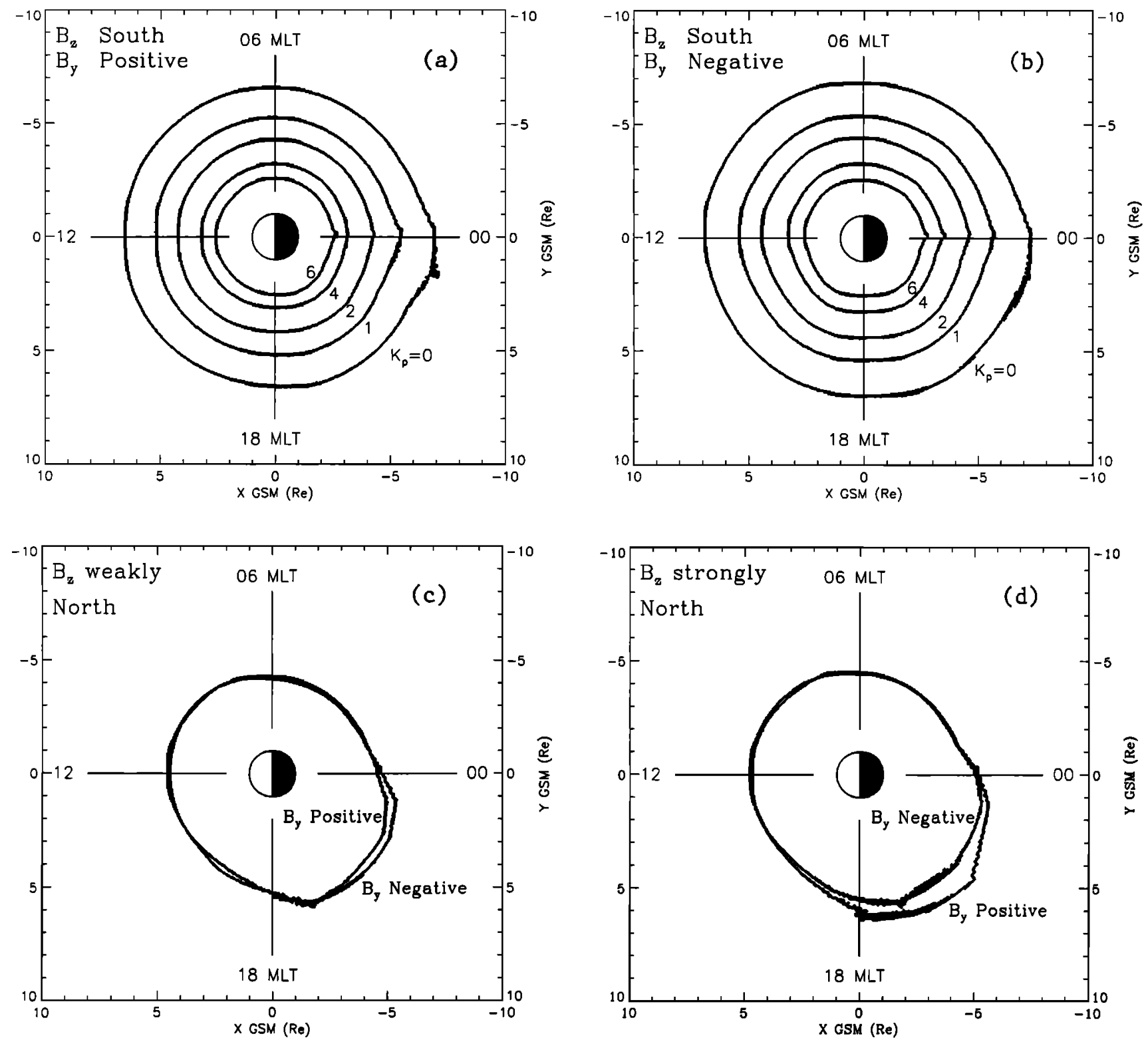

Fig. 4. (a) The plasmapause shapes for IMF $B_{z}$ southward with different Kp values for IMF $B_{y}$ positive derived from our potential mapping technique. (b) Same as Figure $4 a$ except for IMF $B_{y}$ negative. (c) The effect of IMF $B_{y}$ on the morphology and size of the plasmapause for IMF $B_{z}$ weakly northward. (d) The effect of IMF $B_{y}$ on the morphology and size of the plasmapause for IMF $B_{z}$ strongly northward. Fig. 5. The position of the plasmapause around midnight for different $\mathrm{Kp}$ values. The results of Taylor et al. [1969] are represented by crosses. Carpenter and Park's [1973] data are shown as triangles. Horwitz et al. [1990] sorted their data in broad Kp bins (0-3 and 3-6) which are shown as the horizontal lines. The error bar represents one standard deviation around the third order harmonic fit to their data.

pauses observed by Chappell et al. [1972] and Horwitz et al. [1990] and are very different from the circular plasmapauses given by the Heppner and Maynard convection patterns for IMF $B_{z}$ southward. Therefore it seems the IMF $B_{z}$ direction has a marked effect on the size and shape of the plasmapause and could be the reason for the differences in plasmapause morphology presented in the earlier studies for quiet (low $\mathrm{Kp}$ ) conditions.

\section{Analytic Fit to Model Results}

The overall similarity of the IMF $B_{z}$ southward plasmapause shapes shown in Figures $4 a$ and $4 b$ suggests that a single $K_{p}$ dependent model can predict the average plasmapause radius. A simple exponential can fit the shapes to within $5 \%$ and is given for $B_{y}$ positive and $B_{y}$ negative:

$$
\begin{array}{ll}
\text { for } B_{y} \text { positive, } & L_{p p}=(6.14) \exp (-0.156(\mathrm{Kp})) \\
\text { for } B_{y} \text { negative, } & L_{p p}=(6.44) \exp (-0.162(\mathrm{Kp}))
\end{array}
$$

The IMF $B_{z}$ northward oval-shaped plasmapauses can be fit to within $5 \%$ with the following fourth harmonic cosine series:

$$
L_{p p}=L_{0}+\sum_{n=1}^{4} L_{n} \cos \left[\frac{n \pi}{12}\left(\mathrm{LT}+\Phi_{n}\right)\right]
$$


TABLE 1. Analytic fit coefficients for the IMF $B_{z}$ north plasmapause shapes.

\begin{tabular}{cccccccccc}
\hline$I M F$ & $L_{0}$ & $L_{1}$ & $\Phi_{1}$ & $L_{2}$ & $\Phi_{2}$ & $L_{3}$ & $\Phi_{3}$ & $L_{4}$ & $\Phi_{4}$ \\
\hline$B_{z}$ weakly north & & & & & & & & & \\
$\quad \begin{array}{l}B_{y}>0 \\
B_{y}<0\end{array}$ & 4.73 & 0.557 & 4.75 & 0.394 & 7.29 & 0.136 & 11.7 & 0.027 & 8.83 \\
$B_{z}$ strongly north & 4.76 & 0.738 & 4.43 & 0.518 & 7.21 & 0.125 & 11.9 & 0.088 & 0.12 \\
$B_{y}>0$ & 5.10 & 0.848 & 4.40 & 0.561 & 7.03 & 0.183 & 10.9 & 0.015 & 9.63 \\
$B_{y}<0$ & 4.88 & 0.544 & 4.60 & 0.356 & 6.82 & 0.088 & 9.18 & 0.060 & 0.44 \\
\hline
\end{tabular}

where $L_{0}$ is the average radius, $L_{n}$ is the harmonic amplitude, LT is the local time, $\Phi_{n}$ is the harmonic phase shift in units of hours, and $L_{p p}$ is the distance to the plasmapause in Earth radii. Table 1 summarizes all the required coefficients.

\section{Effect of Geomagnetic Activity on the Plasmapause}

The size of the plasmasphere shrinks as magnetospheric convection becomes stronger. Figures $4 a$ and $4 b$ demonstrate this effect for the $B_{z}$ south Heppner and Maynard model. Plotted is the last closed equipotential line for different Kp levels. For both IMF $B_{y}$ positive and $B_{y}$ negative, the largest changes in plasmapause position $\left(L_{p p}\right)$ occur in the dusk sector and range from $L=3$ to $L=7$ for $\mathrm{Kp}=$ 6 to $K p=0$, respectively. Note the virtual lack of a bulge region for all values of $K_{p}$ for these "equipotential plasmapauses." Carpenter [1970] also observed the disappearance of the bulge, but the lack of a bulge was correlated with periods of prolonged quiet.

A summary plot of the midnight plasmapause distance versus $\mathrm{Kp}$ for the two different IMF $B_{y}$ directions is shown in Figure 5. The results of Taylor et al. [1969], who measured $\mathrm{H}^{+}$densities at high altitudes to identify the light ion trough, are plotted as a comparative indication of the position of

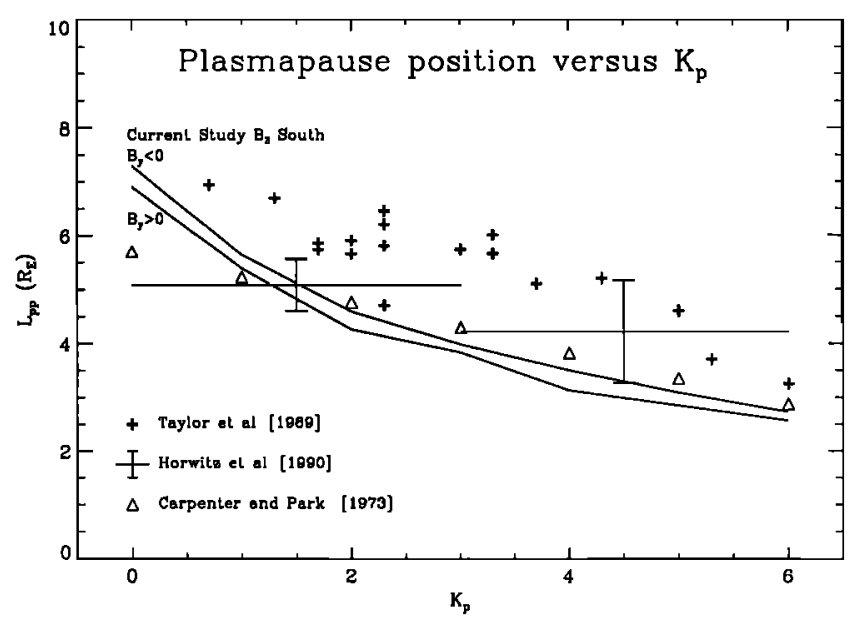

Fig. 5. The position of the plasmapause around midnight for different Kp values. The results of Taylor et al. [1969] are represented by crosses. Carpenter and Park's [1973] data are shown as triangles. Horwitz et al. [1990] sorted their data in broad $K_{p}$ bins (0-3 and 3-6) which are shown as the horizontal lines. The error bar represents one standard deviation around the third order harmonic fit to their data. the plasmapause. Also shown in Figure 5 is the empirically determined size of the plasmapause in the midnight-dawn sector determined by Carpenter and Park [1973]. They characterized their results by the following relationship:

$$
L_{p p}=5.7-0.47(\mathrm{Kp})
$$

The triangles in Figure 5 correspond to the results of the above equation. Also shown are the results of Horwitz et al. [1990], who used the DE 1 retarding ion mass spectrometer (RIMS) instrument to determine the position of the plasmapause by examining the transition from a cold and isotropic ion population to a hot and field-aligned population. The DE RIMS data were binned into two ranges of $K_{p}$ corresponding to $K p=0-3$ and 4-6. The vertical error bar corresponds to one standard deviation about a third harmonic fit to the data. The results of our study lie close to but below the Taylor et al. data and within the fitted error of the Horwitz et al. data. The Carpenter and Park empirical model results compare well to our model results at all $\mathrm{Kp}$, except our model predicts larger plasmapauses for $K p=0$. These differences may be a selection effect due to lack of observations during periods of prolonged geomagnetic quiescence. Taylor et al. used the maximum value of $K p$ in the previous 24 hours to organize their satellite observations. Therefore the values of Kp used in their study are greater than or equal to the actual 3-hour Kp at the time of their measurements. Eliminating this bias would move their data points over to the left of Figure 5 and closer to the results from this current study.

The implications of the MLT location of the bulge with respect to the penetration of the magnetospheric convection electric field have been discussed by several authors (see, for example, Maynard and Grebowsky [1977] and Carpenter [1970]). Maynard and Grebowsky [1977] assume that the farther into the dayside that magnetospheric convection penetrates (the signature being the appearance of the bulge), the less effect the ring current has on shielding the magnetospheric convection electric field. Using Explorer $45 \mathrm{dc}$ probe data, they found the bulge to lie statistically in the evening sector. They therefore assumed that the ring current effectively shields the magnetospheric electric field from the dayside. The results of our study show that the magnetospheric convection electric field is only able to penetrate into the evening sector for IMF $B_{z}$ north conditions.

The circular IMF $B_{z}$ south plasmapauses show little, if any, evidence of bulge movement with increasing Kp. Carpenter [1970] showed that the dusk-side bulge can be located between 1300 and 2100 LT and rotates significantly away 
from noon as $\mathrm{Kp}$ is decreased. The large bulge at $2100 \mathrm{LT}$ for the IMF $B_{z}$ north conditions, presumably corresponding to low $\mathrm{Kp}$ values, agrees with this specific observation. We again emphasize the limitations of using a steady state method during periods of rapidly changing geomagnetic activity.

\section{CONCLUSIONS}

This study tested the concept of using empirical ionospheric convection models as a diagnostic for determining the equatorial position of the plasmapause under conditions of steady geomagnetic activity. The Heppner and Maynard [1987] model was found to be best suited for this pilot study due to the geophysically meaningful input parameters, namely IMF direction and $\mathrm{Kp}$ index.

The main results of this study are as follows:

1. The determination of the location and shape of the plasmapause can be determined using the high-latitude convection model of Heppner and Maynard. The results obtained from mapping the polar potential field to the equatorial plane are consistent with the shape and behavior observed by ground-based and satellite studies of the plasmapause [Carpenter, 1970; Chappell et al., 1970; Maynard and Grebowsky, 1977; and Horwitz et al., 1990].

2. The size of the plasmapause ranges from $L=3$ to $L=$ 7 and varies with geomagnetic activity, the largest plasmasphere corresponding to quiet conditions.

3. The characteristic plasmapause shape is strongly dependent on IMF $B_{z}$ direction, with circular plasmapauses found with IMF $B_{z}$ southward and oval plasmapauses for IMF $B_{z}$ northward.

4. The plasmapause bulge is located at 2100 MLT for IMF $B_{z}$ northward.

This is the first study to examine the effect of IMF direction on the size, shape, and behavior of the plasmapause. Yet, we are hindered by the lack of direct measurements of the plasmapause by spacecraft at all local times under varying IMF conditions. All prior plasmapause observations were not organized into statistical studies using IMF $B_{z}$ as a parameter. This could possibly explain the discrepancies between the four studies shown in Figure 2. The previous observations could be reexamined using IMF $B_{z}$ direction as a binning parameter to examine this possibility. The CRRES mission launched during the summer of 1990 was designed in part to make plasmapause measurements. A study of plasmapause behavior with respect to IMF can then be made and the results compared to the features described in the present model. The model itself could be improved by the availability of input ionospheric convection patterns that emphasize mid-latitude measurements and IMF dependence.

Acknowledgments. This study was performed in partial fulfillment of the requirements for M. Mendillo's Astronomy 776 SolarTerrestrial Physics course at Boston University spring 1990. We thank Fred Rich at AFGL for providing the Heppner and Maynard code. We thank our classmates and also George Siscoe, Jeffrey Hughes, Henry Rishbeth, Gary Erickson and Donald Carpenter for their helpful comments.

The Editor thanks J. L. Horwitz and another referee for their assistance in evaluating this paper.

\section{REFERENCES}

Brice, N. M., Bulk motion of the magnetosphere, J. Geophys. Res., $72,5193,1967$.

Carpenter, D. L., Whistler studies of the plasmapause in the magnetosphere, 1, Temporal variations in the position of the knee and some evidence on plasma motions near the knee, J. Geophys. Res., 71, 693, 1966.

Carpenter, D. L., Whistler evidence of the dynamic behavior of the duskside bulge in the plasmasphere, J. Geophys. Res., 75, $3837,1970$.

Carpenter, D. L. and C. G. Park, On what ionospheric workers should know about the plasmapause-plasmasphere, Rev. Geophys., 11, 133, 1973.

Chappell, C. R., K. K. Harris, and G. W. Sharp, The morphology of the bulge region of the plasmasphere, J. Geophys. Res., 75, $3848,1970$.

Chappell, C. R., K. K. Harris, and G. W. Sharp, The dayside of the plasmasphere, J. Geophys. Res., 76, 7632, 1971.

Chen, A. J., and R. A. Wolf, Effects on the plasmasphere of a time-varying convection electric field, Planet. Space Sci., 20, $483,1972$.

Erickson, G. M., R. W. Spiro, and R. A. Wolf, The physics of the Harang discontinuity, J. Geophys. Res., 96, 1633, 1991.

Grebowsky, J. M., Model study of plasmapause motion, J. Geophys. Res., 75, 4329, 1970.

Heelis, R. A., J. K. Lowell, and R. W. Spiro, High-latitude ionospheric convection model, J. Geophys. Res., 87, 6339, 1982.

Heppner, J. P., and N. C. Maynard, Empirical high latitude electric field models, J. Geophys. Res., 92, 4467, 1987.

Holt, J. M., R. H. Wand, J. V. Evans, and W. L. Oliver, Empirical models for plasma convection at high latitudes from Millstone Hill observations, J. Geophys. Res., 92, 203, 1987.

Hones, A., and J. E. Bergeson, Electric field generated by a rotating magnetized sphere, J. Geophys. Res., 70, 4851, 1965.

Horwitz, J. L., R. H. Comfort, and C. R. Chappell, A statistical characterization of plasmasphere density structure and boundary locations, J. Geophys. Res., 95, 7937, 1990.

Maynard, N. C., and J. M. Grebowsky, The plasmapause revisited, J. Geophys. Res., 82, 1591, 1977.

Mendillo, M., and M. Papagiannis, Estimate of the dependence of the magnetospheric electric field on the velocity of the solar wind, J. Geophys. Res., 76, 6939, 1971.

Nishida, A., Formation of plasmapause, or magnetospheric plasma knee, by the combined action of magnetospheric convection and plasma escape from the tail, J. Geophys. Res., 71, 5669, 1966.

Park, C. G., Whistler observations of the interchange of ionization between the ionosphere and the protonosphere, J. Geophys. Res., 75, 4249, 1970.

Reif, P. H., R. W. Spiro, and T. W. Hill, Dependence of polar cap potential drop on interplanetary parameters, $J$. Geophys. Res., 86, 7639, 1981.

Southwood, D.J., and R.A. Wolf, An assessment of the role of precipitation in magnetospheric convection, J. Geophys. Res., 83, 5227. 1978.

Taylor, H. A., Jr., H. C. Brinton, and M. W. Pharo III, Evidence of contraction of the Earth's thermal plasmasphere subsequent to the solar flare events of 7 and 9 July 1966, Ann. IQSY, 3, $389,1969$.

R. A. Doe, M. Mendillo, and M. B. Moldwin, Center for Space Physics, Boston University, Boston, MA 02215.

(Received February 27, 1991; revised June 17, 1991; accepted June 18, 1991.) 